\title{
Insight into the Molecular Model in Carbon Dots through Experimental and Theoretical Analysis of Citrazinic Acid in Aqueous Solution
}

Antonio Cappai, Claudio Melis, Luigi Stagi, Pier C. Ricci, Francesca Mocci, and Carlo M. Carbonaro*

Cite This: J. Phys. Chem. C 2021, 125, 4836-4845

Read Online

ACCESS

Џlll Metrics \& More

回国 Article Recommendations

S1 Supporting Information

ABSTRACT: The molecular emission model is the most accredited one to explain the emission properties of carbon dots (CDs) in a lowtemperature bottom-up synthesis approach. In the case of citric acid and urea, the formation of a citrazinic acid (CZA) single monomer and oligomers is expected to affect the optical properties of the CDs. It is therefore mandatory to elucidate the possible role of weak bonding interactions in determining the UV absorption spectrum of some molecular aggregates of CZA. Although this carboxylic acid is largely exploited in the synthesis of luminescent CDs, a full understanding of its role in determining the final emission spectra of the produced CDs is still very far to be achieved. To this aim, by relying on purely firstprinciples density functional theory calculations combined with

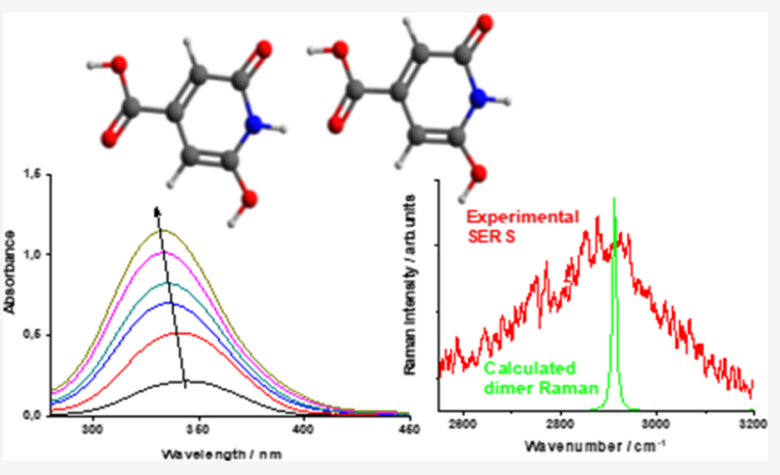
experimental optical characterization, we built and checked the stability of some molecular aggregates, which could possibly arise from the formation of oligomers of CZA, mainly dimers, trimers, and some selected tetramers. The computed vibrational fingerprint of the formation of aggregates is confirmed by surface-enhanced Raman spectroscopy. The comparison of experimental data with calculated UV absorption spectra showed a clear impact of the final morphology of the aggregates on the position of the main peaks in the UV spectra, with particular regard to the $340 \mathrm{~nm}$ peak associated with $\mathrm{n}-\pi^{*}$ transition.

\section{INTRODUCTION}

Since their discovery in $2004,{ }^{1}$ light-emitting carbon dots (CDs) have attracted great interest in the scientific community: they have been proposed, in fact, as promising candidates for the realization of a new generation of lightemitters, ${ }^{2}$ biomarkers, ${ }^{3-5}$ sensors, ${ }^{6,7}$ and photocatalysts. ${ }^{8,9}$ Taking into account the low-temperature bottom-up approach, a complete understanding of the formation mechanism of CDs is still missing because of the involvement of a complex series of chemical steps starting from the reaction and occasionally the polymerization of the precursors, the formation of aggregates, and eventually the carbonization of the macromolecules. The formation of graphitic structures or the presence of aromatic domains within the carbon nanoparticles was largely reported in the literature, calling for a molecule-like model to explain the photophysical properties of CDs. ${ }^{10-13}$

There are mainly three different emission mechanisms to explain the observed optical properties, and in particular, the excitation-dependent emission: (i) the core emission, ascribed to the quantum confinement effect or to conjugated $\pi$-domains within the core of CDs, (ii) the surface states, where the emission features are assigned to surface functional groups bonded to the carbon backbone, and (iii) the molecular state, where fluorescent molecules, free or bonded to the $\mathrm{CD}$ structure, are responsible for the gathered emission. ${ }^{10-13}$ The molecular model was proven to be successful by quantomechanical calculations where different polyaromatic hydrocarbons (PAHs) were exploited to simulate the optical properties of CDs. ${ }^{14-16}$ In particular, it was reported that PAHs can accurately reproduce the peculiar excitationdependent emission of CDs. In some papers, the formation of aggregates (typically dimers) $)^{17-20}$ or multiple layers ${ }^{21}$ was considered the leading mechanism to explain the optical features. Very recently, the formation of fluorescent stacked dimers of imidazo[1,2-a]pyridine-7-carboxylic acid (IPCA) was theoretically investigated as a model for molecular luminescent centers in CDs. ${ }^{22}$ Indeed, by molecular dynamics and quantum-chemistry calculations, it was shown that the aggregates can be embedded within the $\mathrm{CD}$ structure or bonded at the surface, in both cases preserving their optical features. It was also proposed that the control of the

Received: December 7, 2020

Revised: February 9, 2021

Published: February 19, 2021 
interaction among emitting surface centers, such as perylene, is an efficient strategy to tune the emission down to bright red color. $^{23}$ One of the main characteristics of the bottom-up approach is that the intermediate macromolecules and sometimes also the precursors are fluorescent species. ${ }^{24,25}$ Indeed, this represents an internal difficulty of the approach because several intermediates and byproducts formed during the synthesis display emission features in a range of frequencies very close to the ones of the desired $\mathrm{CD} .^{26,27}$ Among the precursors, citric acid and urea are, by far, the most exploited ones, the nitrogen atoms being requested to enhance the quantum yield of the final nanoparticles. ${ }^{28,29}$ As expected, citrazinic acid (CZA) was found to be present during the formation of CDs in the synthesis using citric acid and urea. $^{30,31}$ Even if CZA is widely used to synthesize a whole class of organic molecules proposed as the building block of new optoelectronic devices, a complete understanding of the optical properties of this reactant is still far to be achieved. In particular, besides few papers reported in the literature for the IR region, ${ }^{32}$ the effect of the formation of oligomers, such as dimers, trimers, and tetramers, a common feature of carboxylic acids, on the intensities and position of the absorption spectral lines has not been rationalized yet using a quantum mechanical (QM) computational approach. Notably, QM theoretical investigations are almost absent for the $\mathrm{UV} /$ visible (UV/vis) region. ${ }^{20}$ Even though our calculations are specifically referred to free molecules and the formation of their aggregates in solution, we believe that, according to the cited literature, ${ }^{22}$ those aggregates could be relevant also for the case of bonded and embedded molecular species in CDs.

The lack of a fully fledged theoretical investigation can be ascribed to the intrinsic difficulties in computing noncovalent bonding interactions, such as hydrogen bonds and Van der Waals forces responsible for the dimerization, which are in general very complex to be modeled in a purely quantum chemical computational scheme. ${ }^{33}$ The recent progress in the parametrization of density functional theory (DFT) functionals, however, can nowadays allow numerically treating this kind of interaction by means of the so-called long-range corrected functionals, as the $\omega \mathrm{B} 97 \mathrm{XD} .{ }^{34-36}$ By means of this new class of functionals, it is now possible to correctly describe the effect of hydrogen bonds not only on geometries ${ }^{37}$ but also on the absorption UV/vis spectral lines, which are, in turn, affected by the formation of aggregates.

In this combined computational-experimental study, we will focus on the identification and optical characterization of several possible dimers, trimers, and some selected tetramers of the CZA in water solution. In particular, by means of a purely DFT approach, we identified the most stable configuration arising from hydrogen bonding of two, three, and four CZA molecules. The effect of the resulting geometries on the position and intensity of the UV absorption peaks is calculated and compared to experimental data and their interpretation within the excitonic theory framework. ${ }^{29,38,39}$ Computed vibrational features of aggregates are also in very good agreement with experimental surface-enhanced Raman spectroscopy (SERS) measurements, here reported for the first time. These results offer a realistic interpretation of optical features recorded in CDs, supporting the molecular model and the formation of aggregates as one of the main mechanisms for the observed absorption and emission properties.

\section{EXPERIMENTAL AND COMPUTATIONAL METHODS}

CZA (purity 0.97, Sigma-Aldrich) and milli-Q water were used as received without further purification. Different solutions of CZA in water were prepared with concentrations in the $0.01-$ $110 \mathrm{mg} / \mathrm{L}$ range. The absorbance spectra in the UV-vis range of CZA solubilized in water were recorded with a Nicolet Evolution 300 spectrophotometer from 200 to $600 \mathrm{~nm}$ (cuvette optical path: $10 \mathrm{~mm}$ ).

Micro-Raman scattering measurements were performed in backscattering geometry through the confocal system SOL Confotec MR750 equipped with a Nikon Eclipse Ni microscope, exciting the samples at $532 \mathrm{~nm}$ (IO Match-Box series laser diode). Spectral resolution was $0.6 \mathrm{~cm}^{-1}$. SERS supports were ITO glasses coated with silver nanoparticles (SSilver SERS substrates, Sersitive, Warsaw Poland).

All the quantum-chemistry calculations were performed using the Gaussian 16 suite of programs. ${ }^{40}$ We assumed as starting geometry the keto tautomer of CZA which, from preliminary calculation, was more stable in water solution than the imine tautomer.

We performed a geometry optimization down to the selfconsistent field (SCF) energy of the monomer by means of DFT calculations carried out at a B3LYP/6311++G(d,p $)^{41,42}$ and $\omega \mathrm{B} 97 \mathrm{XD} / 6-311++\mathrm{G}(\mathrm{d}, \mathrm{p})^{34-36}$ level of theory. Solvation effects arising from the interaction of CZA molecules with water used as the solvent were treated with the self-consistent reaction field model by simulating the dielectric solvent through the polarizable continuum model calculation within the integral equation formalism (IEFPCM). ${ }^{43}$

UV absorption spectra were simulated in terms of vertical energy transitions with the solvent environment assumed to be the same of the ground state of the molecule using both the functionals with the same basis set. All the structures were verified to be real energy minima with no imaginary frequencies in the vibrational spectra. This protocol was successively applied with all the aggregates generated as described in the next paragraph.

Starting from the optimized geometry of the monomer, we built the following dimer configurations, as depicted in Figure 1:

1 stack-parallel. The two monomers are assumed lying in two parallel planes at a variable distance $3 \AA \leq \mathrm{z} \leq 5 \AA$;

2 rot-z. Starting from a geometry generated in case 1 , a subsequent rotation $\theta$ along the $\mathrm{z}$ axis is applied;

3 rot-y. Initially, two monomers are supposed to lie in the same $\mathrm{z}=0$ plane with the nitrogen atom of a monomer facing the carboxyl group of the other. A rotation $\varphi$ is then applied along the nitrogen-nitrogen axis (assumed as $\mathrm{y}$ axis);

4 flipped-rot-y. As in case 3 but starting with two monomers facing each other with the nitrogen atoms.

5 tile rotation. Starting from the geometry obtained in case 3 with $\varphi=0$, the system is then modified by increasing the dihedral angle determined by the two monomers. The stability of the optimized starting geometry was also studied by rotating the dimers around the major and minor axes of the dimeric system.

\section{RESULTS AND DISCUSSION}

3.1. Assessing the Functional Validity in Describing Nonbonding Interactions. To check the validity of the 


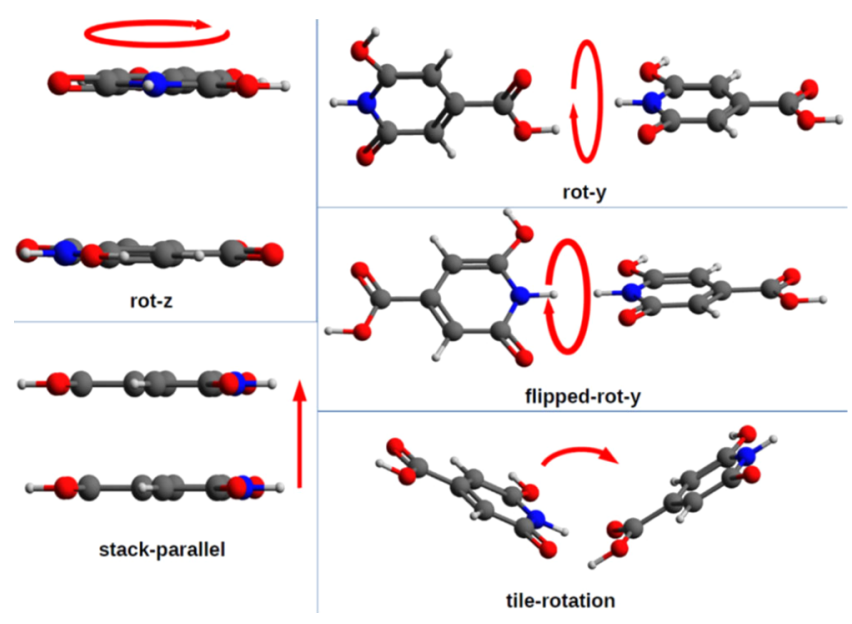

Figure 1. Ball-and-stick representation of the five starting dimer configurations. Red arrows indicate the direction of translation and rotation. (white sphere $=\mathrm{H}$ atom, gray sphere $=\mathrm{C}$ atom, red sphere $=$ $\mathrm{O}$ atom, and blue sphere $=\mathrm{N}$ atom $)$.

B3LYP and $\omega$ B97XD functionals in describing nonbonding interactions responsible for the dimerization of the CZA in solution, we defined the binding energy of the system as

$$
\Delta E=E_{\text {dimer }}-2 E_{\text {monomer }}
$$

where $E_{\text {dimer }}$ represents the total DFT SCF energy resulting from the optimization of the entire dimeric system while $E_{\text {monomer }}$ is the corresponding energy of an isolated monomer calculated at the same level of theory.

As it is well known that the energy of aggregate systems can be affected by a basis set superposition error (BSSE), a separate calculation was performed to quantify the amount of the correction to be applied and obtain an accurate estimate of the binding energy (see the Supporting Information for details): using both functionals, a total BSSE correction of 1.4 $\mathrm{kcal} \mathrm{mol}^{-1}$ was estimated leading to a complexation energy of $+7.63 \mathrm{kcal} \mathrm{mol}^{-1}$ in the case of the B3LYP functional and $1.02 \mathrm{kcal} \mathrm{mol}^{-1}$ in the case of the $\omega \mathrm{B} 97 \mathrm{XD}$ functional. The correction was then calculated and applied to the total SCF binding energies of all the structures (see Table 1).

Table 1. Sample ID and Total DFT SCF Binding Energies, Calculated before and after the Corrections Accounting for BSSE for the Six Dimeric Systems Analyzed

\begin{tabular}{clcc}
$\begin{array}{c}\text { sample } \\
\text { ID }\end{array}$ & $\begin{array}{c}\text { configuration } \\
\text { dimer-1 }\end{array}$ & $\begin{array}{c}\text { hCF energy } \\
\left.\mathrm{mol}^{-1}\right)\end{array}$ & $\begin{array}{c}\mathrm{BSSE} \text { corrected energy } \\
\left(\mathrm{kcal} \mathrm{mol}^{-1}\right)\end{array}$ \\
dimer-2 & tail-to-tail & -8.837 & -7.890 \\
dimer-3 & head-to-tail & -10.391 & -9.495 \\
dimer-4 & symmetric-stack- & -13.251 & -12.327 \\
& $\begin{array}{c}\text { parallel } \\
\text { dimer-5 }\end{array}$ & -7.798 & -5.423 \\
dimer-6 & $\begin{array}{l}\text { mirror-stack- } \\
\text { parallel }\end{array}$ & -4.057 & -1.899 \\
& -1.541 & -0.0039 \\
\hline
\end{tabular}

As reported in the Supporting Information (Figure S1), by a direct analysis of the results obtained for the stack-parallel configuration, it emerges that $\omega \mathrm{B} 97 \mathrm{XD}$ can clearly identify the presence of a stable dimer aggregate, while in the case of the B3LYP functional, no stable configurations can be found for any $\mathrm{z}$ stacking distance. In the case of the planar dimer configurations (vide infra), considering the representative head-to-tail dimer, we obtained the formation of an energy minimum with both the functionals at about the same distance of the two monomer units, the energy minimum being about $2-3 \mathrm{kcal} / \mathrm{mol}$ lower for the $\omega \mathrm{B} 97 \mathrm{XD}$ case than for the B3LYP one. These results indicate that the $\omega \mathrm{B} 97 \mathrm{XD}$ functional must be considered to calculate all possible dimers to explain the experimental data that suggest the occurrence of aggregated structures in aqueous solutions. ${ }^{21}$

Taking into account the observed ability to reproduce aggregation phenomena, for further analysis we assumed the long-range corrected $\omega \mathrm{B} 97 \mathrm{XD}$ functional for geometrical optimization of all the structures of interest. The gathered results are the following: (i) none of the rot-y and flipped-rot-y configurations provide an energy minimum; (ii) the stackparallel configuration is stable for a stacking distance of $\mathrm{z}=$ $3.66 \AA$ with a binding energy of $-0.039 \mathrm{kcal} \mathrm{mol}^{-1}$; (iii) a minimum energy configuration was retrieved for the tile rotation dimer only at $\theta=0^{\circ}$, and the energy of the dimer keeps increasing as the dihedral angle among dimers increases; and (iv) the rot-z configuration is stable $\left(-1.899 \mathrm{kcal} \mathrm{mol}^{-1}\right)$ for two (symmetric) values of the rotation angle $\theta$ corresponding to $\theta=54$ and $306^{\circ}$ at a distance of $\mathrm{z}=3.25$ $\AA$. Thus, the stack-parallel and rot-z models were further considered in the discussion, together with three more planar configurations suggested by the electrostatic potential map of the monomer (vide infra).

3.2. Stability Analysis of Dimers and Electrostatic Potential Map. Using the Gaussian 16 suite of programs, the electrostatic potential map for the CZA monomer was calculated at the $\omega \mathrm{B} 97 \mathrm{XD} / 6-311++\mathrm{G}(\mathrm{d}, \mathrm{p})$ level of theory: the result, reported in Figure 2, evidences, as expected, a high electronic charge density on the ketone oxygen and a moderate nucleophilic potential on the carboxylic oxygen, while one of the highest and dense electrophilic potentials is observed in correspondence with the pyridinic nitrogen.

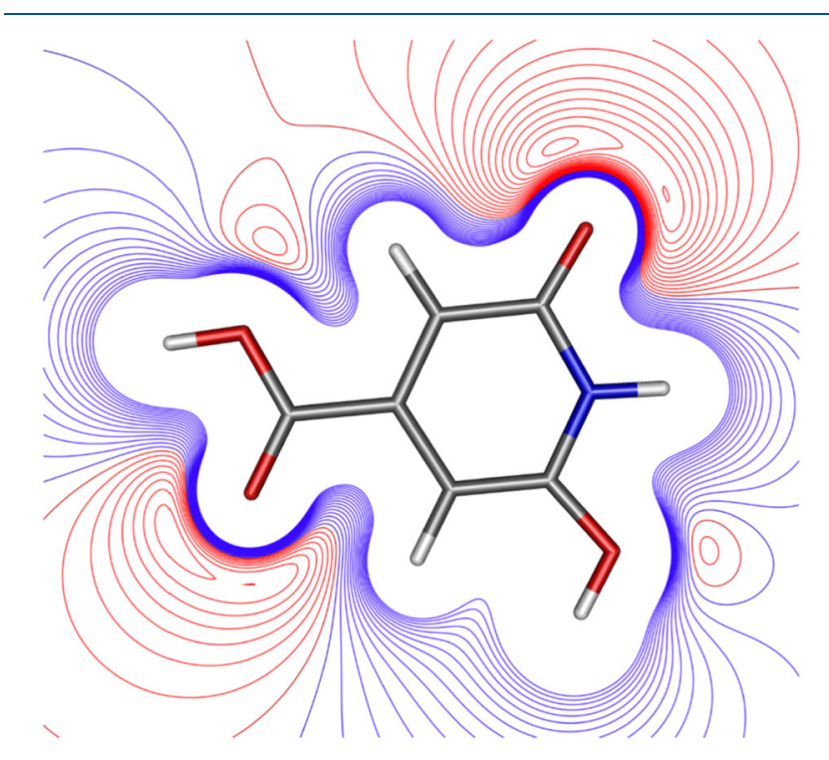

Figure 2. Molecular electrostatic potential for an isolated CZA molecule in aqueous solution. The map displays isovalue contours in the range $[-0.1: 0.1]$ electrostatic potential in atomic units in the molecular plane: red regions indicate negative potentials (nucleophilic-donor coordination sites) and blue regions positive electrostatic potentials (electrophilic-acceptor coordination sites). 
Based on this result, we designed three new dimeric planar systems not evaluated in the previous subsection: a sketch of geometries and main structural parameters of the final six analyzed dimer systems are depicted in Figure 3. As reported,

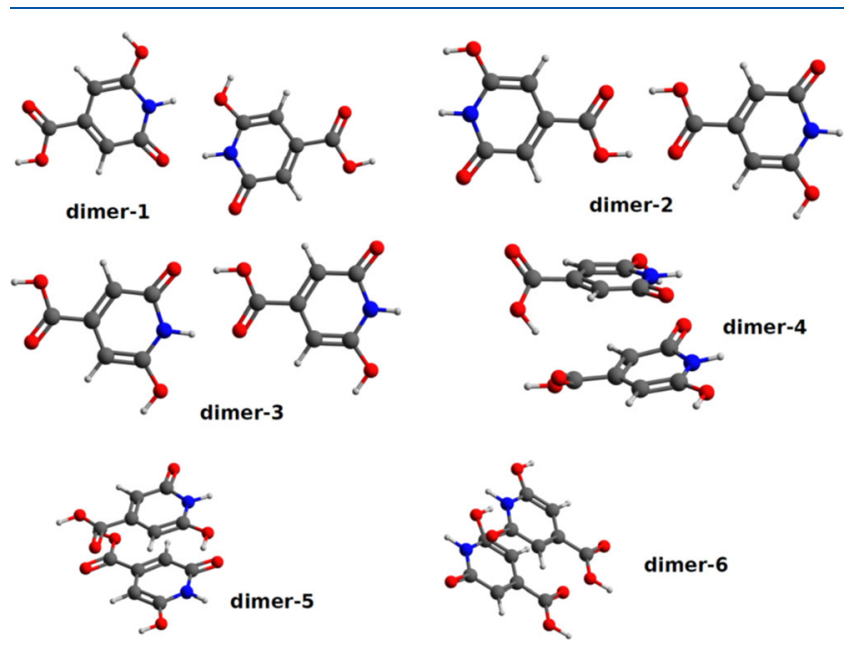

Figure 3. Ball-and-stick representation of the selected dimeric systems: binding energies and configuration descriptions are listed in Table 1 (white sphere $=\mathrm{H}$ atom, gray sphere $=\mathrm{C}$ atom, red sphere $=\mathrm{O}$ atom, and blue sphere $=\mathrm{N}$ atom) .

dimer-1, -2 , and -3 correspond to head-to-head, tail-to-tail, and head-to-tail configurations. Dimer- $4,-5$, and -6 have the already described symmetric-stack-parallel configuration, the rot-z configuration, and the mirror-stack-parallel configuration. When designing the new systems, we took care of not facing electrostatic charges of the same sign to avoid electrostatic repulsion. For example, in the case of dimer- 1 the electrophilic acceptor pyridinic nitrogen site of one monomer was faced to the nucleophilic donor ketone oxygen site of the other.

To verify the stability in aqueous solution of each investigated system, single-point energies and bonding energies were calculated for each system, evaluating BSSE contributions for each dimer. We observed the highest bonding energy in the dimer-3 configuration, while the dimer-6 configuration displayed the lowest one.

A reasonably good stability is further observed in the case of dimerization mediated by the two carboxyl groups while the stability of dimers bound by a ketonic-to-ketonic interaction is considerably lower (see Table 1).

3.3. Experimental and Theoretical UV/vis Absorption Spectra Analysis. Experimental absorption spectra are reported in Figure 4. As the concentration of CZA increases, the absorption peak around $340 \mathrm{~nm}(3.6 \mathrm{eV})$ undergoes a blue shift, typically ascribed to the formation of oligomers.

To analyze the spectral shift in detail, we performed a spectral deconvolution in the energy space with Gaussian bands. The experimental spectra were fitted with two high energy bands, at about 4.5 and $5.5 \mathrm{eV}$, to account for the far UV contributions, and one or two bands in the $3.0-4.0 \mathrm{eV}$ range to reproduce the spectral features of the $3.6 \mathrm{eV}$ (340 $\mathrm{nm})$ band. The fitting results over the whole spectral range are reported in the Supporting Information (Figure S2).

At very low concentration $(0.01 \mathrm{mg} / \mathrm{L})$, the band at $340 \mathrm{~nm}$ was not detected, its signal starting to be detectable in the solution with $0.1 \mathrm{mg} / \mathrm{L}$ of CZA. At this concentration, the absorption spectrum can be successfully fitted with just one

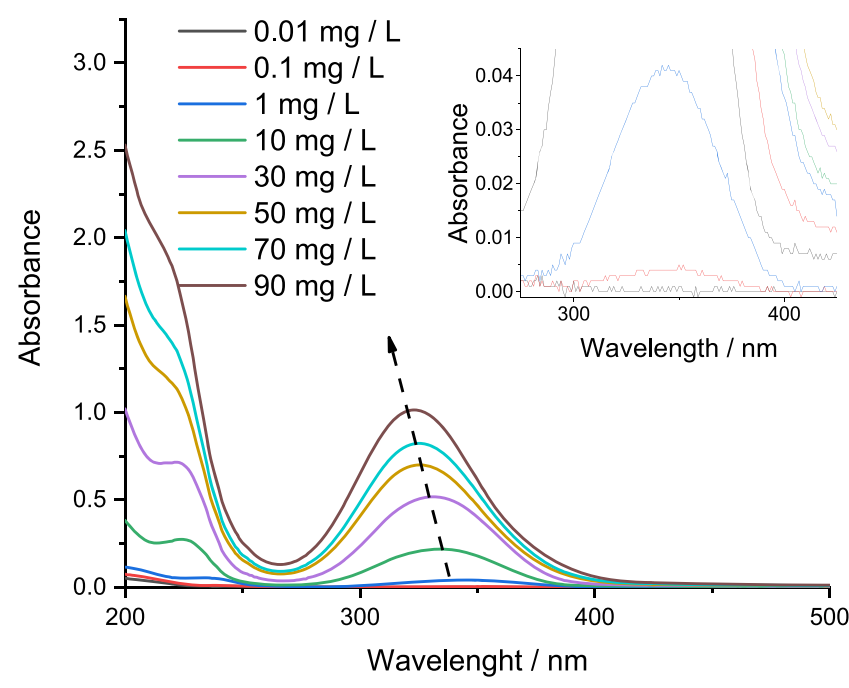

Figure 4. Experimental absorbance spectra as a function of CZA concentration. The inset shows a magnified view of the $300-400 \mathrm{~nm}$ range at low concentration

Gaussian band peaked at $3.63 \mathrm{eV}$ with a full width at half maximum (FWHM) of $0.60 \mathrm{eV}$ (Figure 5).At larger concentration, in the $1-30 \mathrm{mg} / \mathrm{L}$ range, two Gaussian bands are required to fit the spectrum, red and blue shifted with respect to the previous one, at 3.46 and $3.68 \mathrm{eV}$ respectively, with a FHWM of about 0.32 and $0.59 \mathrm{eV}$. Finally, at larger concentrations the band becomes again symmetric, a single Gaussian band at $3.73 \mathrm{eV}$ and FWHM of $0.68 \mathrm{eV}$ being needed to fit the analyzed portion of the absorption spectrum.

The reported data suggest the formation of aggregates of CZA and can be interpreted within the exciton theory framework. ${ }^{38}$ According to the theory, the formation of a composite molecule obtained from the interaction of two single monomers causes a variation in the optical absorption transition energy expressed as follows:

$$
\Delta E_{\text {dimer }}=\Delta E_{\text {monomer }}+\Delta D \pm \Gamma
$$

where $\Delta E$ is the transition energy of the dimer (monomer), $\Delta \mathrm{D}$ is the variation in the Van der Waals contribution in the aggregate structure, and $\Gamma$ is the exciton splitting term. According to the above equation, besides the general lowering of the transition energy because of the Van der Waals interaction, the excited state is split into two energy levels, at higher and lower energy with respect to the monomeric one. These two bands are typically defined as the $\mathrm{H}$ and $\mathrm{J}$ band, respectively, and their observation depends on the spatial arrangement of the two monomer units, which determines if a transition is allowed or not. Indeed, within the exciton theory, there are different possible geometric configurations of the dimers, depending on the alignment of the single unit transition polarization axis, such as parallel and in-line transition dipoles (also known as $\mathrm{H}$ and $\mathrm{J}$ dimers), oblique transition dipoles, and coplanar or nonplanar transition dipoles.

According to this simplified theory, the $\mathrm{H}$ band is the only transition observed in parallel $\mathrm{H}$ dimers, while the $\mathrm{J}$ band is the allowed transition for in-line $\mathrm{J}$ dimers. Both transitions are allowed for the oblique dimers, while for coplanar and nonplanar structures, the energy levels change continuously as a function of the relative orientation of the two monomers. 


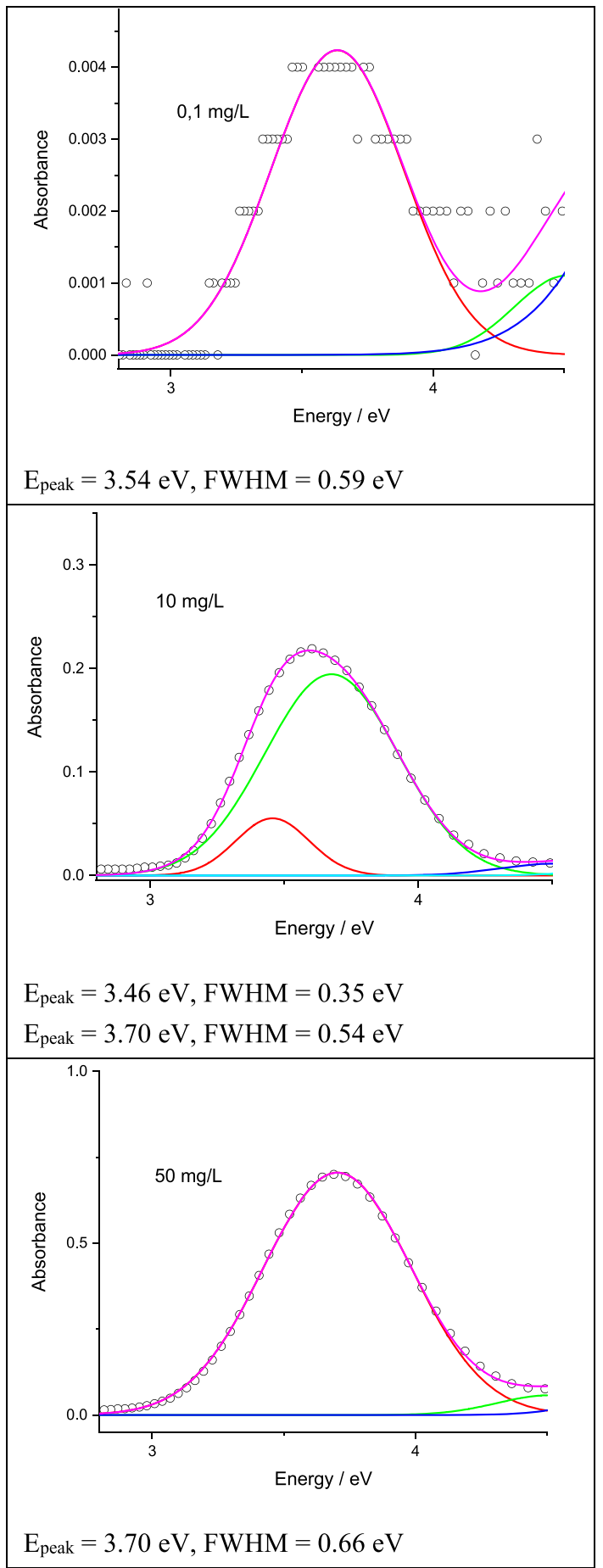

Figure 5. Deconvolution of absorbance spectra at different CZA concentrations with Gaussian bands in the $3.0-4.0 \mathrm{eV}$ range

Within this scenario, the recorded experimental data report the unique formation of the monomeric unit of the molecule (the transition being peaked at $3.63 \mathrm{eV}$ ) at low concentration (below $1 \mathrm{mg} / \mathrm{L}$ ). In the $1-30 \mathrm{mg} / \mathrm{L}$ concentration range, the formation of dimers causes the excitonic splitting of the band, with two transitions recorded at lower and higher energies with respect to the monomeric one $(3.46$ and $3.68 \mathrm{eV}$, respectively, estimated splitting $0.22 \mathrm{eV}$, and Van der Waals interaction $-0.06 \mathrm{eV}$ ).

According to the theory, these findings call for the presence of both $\mathrm{J}$ and $\mathrm{H}$ dimers or the formation of the oblique ones.
As the concentration keeps increasing, a close packing of monomer units is forced, thus $\mathrm{J}$ dimers or oblique ones disappear being transformed into $\mathrm{H}$ dimers. We should, however, keep in mind that the exciton theory is a simplified model that considers only the formation of dimers and how the absorption spectrum changes accordingly as a function of the concentration. The latter, indeed, affects the kind of observed dimers, being the geometrical configuration of the interacting monomeric units related to the surrounding environment. As the concentration increases, however, not only the geometry of the dimers changes, but also larger oligomers, such as trimers and tetramers, can be formed. The theoretical simulations aim to clarify the effect of the presence of various oligomers on the absorption spectrum of CZA solutions.

Theoretical UV/Vis absorption spectra were calculated by means of a time-dependent DFT approach in the framework of Franck-Condon approximation: to assess the validity of our calculated data, a preliminary UV spectrum was generated for the case of the monomer, to be compared to the reported experimental data, using both B3LYP/6-311++G(d,p) and $\omega \mathrm{B} 97 \mathrm{XD} / 6-311++\mathrm{G}(\mathrm{d}, \mathrm{p})$ combination. We obtained in this case that the B3LYP functional can better fit the experimental pattern observed, while the $\omega \mathrm{B} 97 \mathrm{XD}$ functional leads to a substantial underestimation of absorption wavelength. For this reason, the UV/vis spectra of the whole set of investigated structures were calculated with the B3LYP functional.

In Figure 6, UV/vis absorption spectra are depicted for the case of two isolated CZA molecules (labeled as a monomer)

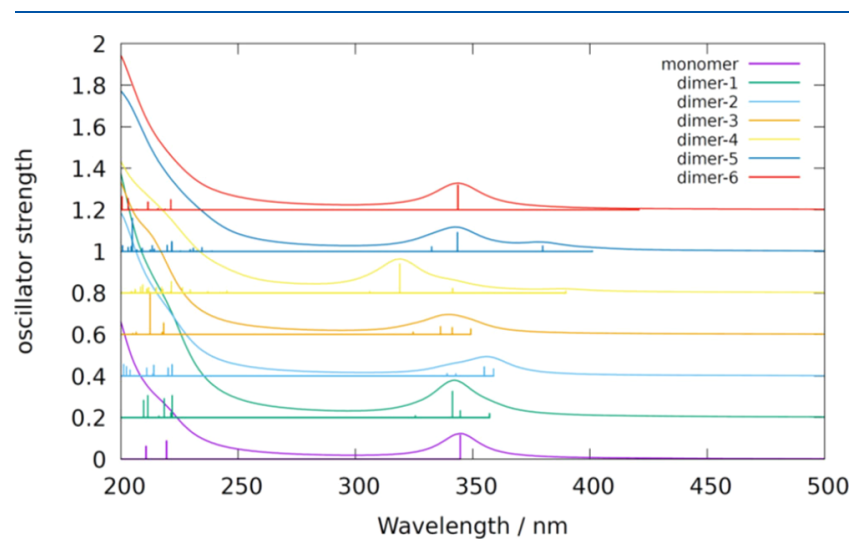

Figure 6. UV/vis absorption spectra simulated for the case of two isolated CZA molecules (labeled as a monomer) and the six dimeric systems depicted in Figure 3 and referenced in Table 1. A constant shift of 0.2 arbitrary units is applied for the sake of comparison.

and the six dimeric system analyzed. To emphasize the observed shift in the absorption peaks, spectra are shown in terms of arbitrary absorbance units where a constant shift of 0.2 units is applied. Referencing to Figure 6, a bathochromic shift is observed in the case of dimer- 2 if compared to the case of two isolated molecules, and a similar effect is observed in dimers 4 and 5 , because of the arising of a previously unobserved absorption peak for $\lambda>344 \mathrm{~nm}$.

In contrast, no significant difference can be found in the case of dimer 6, as expected from the negligible binding energy of the system, a condition which can justify the treatment of dimer 6, from an optical point of view, as two isolated CZA molecules.

The dimer-3 absorption spectrum, finally, evidences a conspicuous broadening of the absorption wavelength range 
centered around $\lambda=344 \mathrm{~nm}$ : this effect is explained by the splitting of the previously unique absorption peak at $\lambda$ into two different, less intense, absorption peaks, symmetrically distributed around $\lambda^{\prime}=340 \mathrm{~nm}$, an effect amenable to the interaction energy between the carboxyl and the pyridinic groups of the two monomers. We point out that solvatochromic studies ${ }^{25}$ reported hypsochromic and bathochromic shifts of the absorption bands in polar protic solvent with respect to polar aprotic ones. Although the experimental and calculated optical properties here reported refer to water solution and differences can be observed in different solvents, water is the typical medium used during the synthesis; thus we believe that the discussed formation of aggregates could be relevant for the general synthesis of CDs.

It is important to underline that the UV spectrum of the most energetically favored dimer, dimer-3, is in very good agreement with the experimental data in the $1-30 \mathrm{mg} / \mathrm{L}$ concentration range, where two excitations were spectrally resolved by means of Gaussian deconvolution. The calculated exciton splitting is in stark agreement with the experimental one; thus we can definitely assign to in-line head-to-tail dimers the measured spectral features. In addition, the formation of other less energetically favored dimers, such as dimer-1 and 2, cannot be ruled out, in particular if we consider the dimerization at the surface or within the core of CDs, where the $\mathrm{C}$ atom network can decrease the formation energy of the process. Those dimers provide a further enlargement of the $340 \mathrm{~nm}$ band, opening new excitation channels down to the near visible range (dimer-1), as in general observed in CDs. It is worth noting that the reported optical properties are in good agreement with the experimental ones reported for CZA aggregates and the related CDs obtained through solvothermal synthesis, ${ }^{26}$ showing by means of transmission electron microscopy (TEM) images that aggregated CZA molecules embedded in a polyvinylpyrrolidone matrix can polymerize and form nanoparticles of about $4 \mathrm{~nm}$. Finally, we point out that the redshifted contributions calculated for the reported dimers agree well with the excitation maximum previously ascribed to fluorescent J-type dimers whose presence can be experimentally monitored through the redshift of the emission peak of citric-acid-related CDs. ${ }^{30}$

3.4. Beyond Dimerization: Trimeric and Tetrameric Aggregates. Using the same approach employed for dimers, three-and four-molecule aggregates were built and analyzed to identify the most stable configuration of these complex structures and verify the impact of possible H-bonded larger structures on the optical activity.

In the construction of the trial configurations, we focused on planar aggregates, and the stability of the aggregate is ensured by the presence of hydrogen bonds because, from the analysis of dimers, the stacking configuration is characterized by a significantly lower interaction energy.

Four possible configurations have been identified for the trimeric system as reported in Figure 7. After optimization using the aforementioned $\omega \mathrm{B} 97 \mathrm{XD}$ functional, the trimer-1 configuration proved to be the most stable and was successively selected for UV-vis absorption spectral analysis.

In a similar fashion, seven tetrameric structures were generated by exploring all the possible orientations of each single monomeric unit, a recursive search being possible thanks to the presence of symmetric constraints (see Figure S3 in the Supporting Information). The most stable structure isolated is depicted in Figure 8.

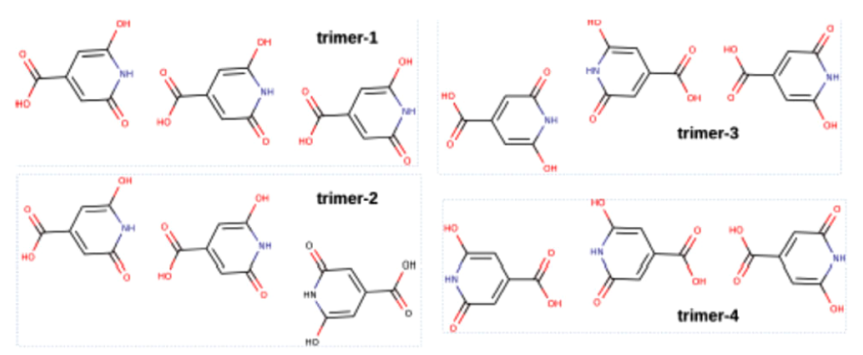

Figure 7. Trial structures for the three-molecule CZA-based aggregates investigated. Trimer-1 configuration emerged as the most stable structure after full DFT geometrical optimization.

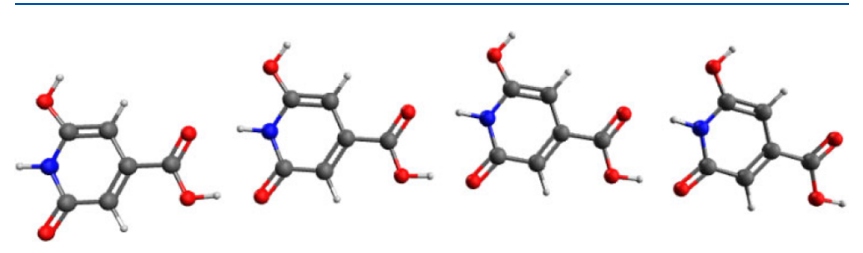

Figure 8. Most energetically stable tetrameric structure.

UV-vis absorption spectra for the most stable two-, three-, and four-molecule aggregates found along with the monomer spectrum as a reference are depicted in Figure 9. Two opposite

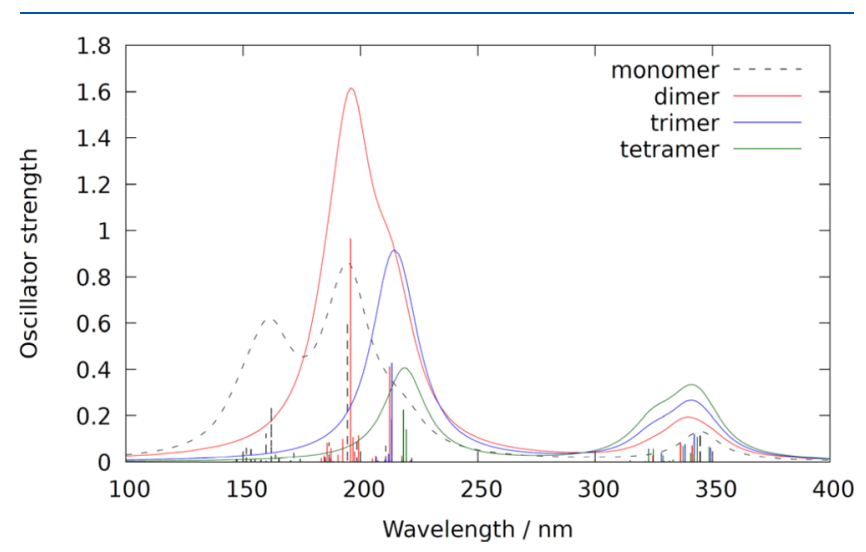

Figure 9. UV-vis spectra for the most stable dimeric (red), trimeric (blue), and tetrameric (dark green) aggregates. The monomer UV spectrum, in a black dashed line, is shown as a reference.

trends emerge in the optical absorbance features of the aggregates as the number of constituent units increases. In the lower wavelength region $(150-250 \mathrm{~nm})$, in fact, a consistent redshift can be traced, with the most prominent modification being visible for the monomer to dimer transition with the suppression of the $\sim 160 \mathrm{~nm}$ peak. As the dimension of the aggregate increases, the main absorption peak at $\sim 190 \mathrm{~nm}$ in the monomer and dimeric system gradually shifts to higher wavelength, positioning at $220 \mathrm{~nm}$ in the tetramer.

In contrast, the increase in dimension of the aggregate produces a blueshift in the higher wavelength region (300-400 $\mathrm{nm}$ ) because of the formation of new excited states, higher in energy compared to the case of the monomer, determining the emergence of a prominent left shoulder around the main absorption line at $\sim 344 \mathrm{~nm}$. Thus, the formation of larger oligomers, expected when increasing the CZA concentration, causes an overall blueshift of the $340 \mathrm{~nm}$ band, as here experimentally observed at concentration larger than $30 \mathrm{mg} / \mathrm{L}$, 
Table 2. Brightest Absorption Transition (Imposing Cut-off on Oscillator Strength $\varepsilon \geq 0.1$ ) for the Most Stable Aggregates of CZA Investigated and Selected for NTO Analysis ${ }^{a}$

\begin{tabular}{|c|c|c|c|c|c|c|c|}
\hline \multicolumn{2}{|c|}{ monomer } & \multicolumn{2}{|c|}{ dimer } & \multicolumn{2}{|c|}{ trimer } & \multicolumn{2}{|c|}{ tetramer } \\
\hline$\lambda(\mathrm{nm})$ & $\varepsilon$ (a.u.) & $\lambda(\mathrm{nm})$ & $\varepsilon$ (a.u.) & $\lambda(\mathrm{nm})$ & $\varepsilon$ (a.u.) & $\lambda(\mathrm{nm})$ & $\varepsilon$ (a.u.) \\
\hline 194.37 & 0.6095 & 195.75 & 0.9637 & 213.32 & 0.4283 & 218.28 & 0.2242 \\
\hline 162.01 & 0.2381 & 212.43 & 0.4125 & 213.17 & 0.1837 & 219.50 & 0.1422 \\
\hline 344.72 & 0.1258 & 218.21 & 0.1154 & 219.51 & 0.1343 & 343.45 & 0.1086 \\
\hline 159.74 & 0.1231 & 199.14 & 0.1149 & 218.16 & 0.1342 & & \\
\hline 198.46 & 0.1051 & 196.83 & 0.1072 & 342.20 & 0.1207 & & \\
\hline
\end{tabular}

${ }^{a_{\text {The }}}$ monomer case is reported as a reference.

and is in general paired to a net decrease of the related emission properties because of quenching phenomena. ${ }^{30,44-46}$

In the perspective of $\mathrm{CD}$ engineering, all the results here reported on the formation of aggregates point out that a strict control on the composition and concentration of precursors should be granted to achieve highly efficient CDs with excitation in the near visible range.

3.5. Natural Transition Orbital (NTO) Analysis. To better clarify the impact of aggregate formation on observed optical spectra, a set of NTOs was generated for each investigated system by performing separate unitary transformations on the occupied and on the virtual set of orbitals to obtain a localized picture of the transition density matrix. ${ }^{47}$

Focusing on the most stable conformations, the transitions to be studied have been selected among the brightest in the two wavelength regions previously pointed out. As a quantitative criterion of selection, a cut-off of 0.1 on the oscillator strength was set, obtaining the ensemble listed in Table 2.

A direct inspection of the numerical values clearly confirms the two trends observed, with a redshift of the main absorption peak in the low-wavelength region from 194 to $218 \mathrm{~nm}$ when aggregate dimension spans from 1 to 4 monomeric units. In the high-wavelength region, instead, no significant shift can be traced for the $344 \mathrm{~nm}$ main peak, confirming that the increase of the blue shoulder observed must be reconnected to the formation of new energy level above the fundamental level characteristic of the monomer.

We observed that all the transitions listed in Table 2 arise from multiple contributions of different molecular orbitals, a circumstance which leads to the use of the NTO to better provide a clearer description of the absorption process in terms of the classical electron-hole framework by a proper localized picture of the transition density matrix, as proposed in Martin's work. $^{47}$

The NTOs for the brightest transitions of the CZA monomer and dimer- 3 in the UV range are reported in Figure 10. The ones for the deep UV range, as well as the trimer and tetramer cases for both ranges, are reported in Figure S3. Within this scenario, the reported absorption at about $340 \mathrm{~nm}$ can be interpreted as because of $n-\pi^{*}$ transitions in the whole set of structures, with the involvement of the $\mathrm{H}$-bond region being observed upon excitation in the aggregates.

As compared to the investigation of IPCA dimers, ${ }^{22}$ there are two issues worth discussing: the geometry of the dimers and the electronic character of the $340 \mathrm{~nm}$ transition. IPCA dimers were proven to aggregate as stacked structures while in the present case we found in-line aggregates. Thus, the formation of different aggregates depending on the bottom-up starting precursors and concentration can explain the nonubiquitous observation of graphitic crystalline planes in CDs.

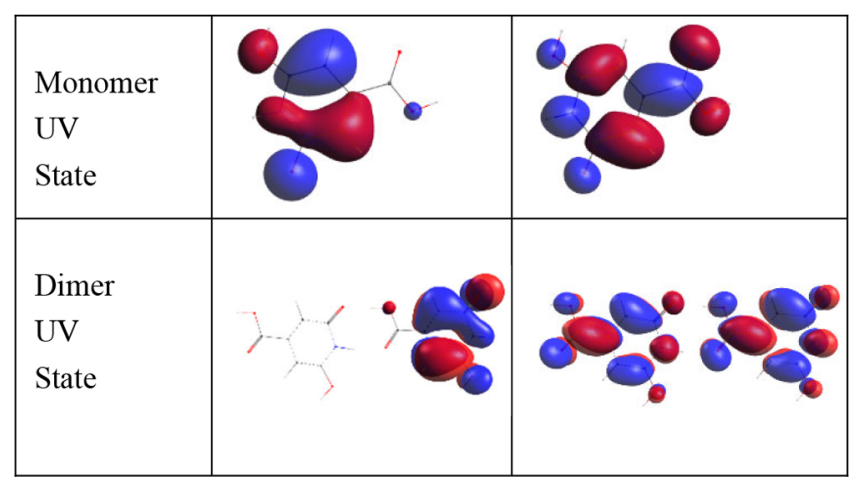

Figure 10. NTOs for the brightest transitions of the CZA monomer and dimer- 3 in the UV range. The isocontour value is $0.02 \mathrm{au}$.

We may speculate that the different geometry could be related to the larger planar configuration of IPCA as compared to CZA.

Second, the reported results for transition orbitals for CZA agree well with the typical $n-\pi^{*}$ character reported for the 340 $\mathrm{nm}$ transition, ${ }^{48}$ while the one calculated for IPCA as well as the previously reported ones for oxygen-functionalized graphitic $\mathrm{CDs}^{49}$ evidenced a $\pi-\pi^{*}$ character. In the latter, the lack of carbonyl groups to which the $n-\pi^{*}$ transition is ascribed, ${ }^{48}$ as also confirmed by our NTO results, can explain the different character of the computed excitation.

As for the aggregates of IPCA, the presence of the imidazole ring leads to a redistribution of the electronic charge upon the five-member cycle thus favoring a $\pi-\pi^{*}$ configuration also in this case.

The simulated Raman and IR spectra of the monomer and aggregates show that all the vibrational features are preserved when the oligomers are formed, with a small blue shift of the calculated modes as the aggregation increases (Figure 11 and Figure S4 in the Supporting Information for the Raman and IR spectra, respectively).

The main signatures of aggregation are the two modes recorded around 2900 and $3200 \mathrm{~cm}^{-1}$ pertaining to the vibrations of the $\mathrm{H}$ atoms bonded to the nitrogen and to the oxygen of the carboxylic group and involved in the H-bonding between two monomers. The reported data agree quite well with previous literature findings (see Supporting Information Table S1), reporting the formation of different tautomers of CZA depending on the $\mathrm{pH}$ of the solution but neglecting the possible presence of oligomers (only reported data refer to 0.1 $\mathrm{M}$ concentration, where, according to our optical absorbance results, aggregates should be expected). Indeed the Fouriertransform infrared spectrum reported by Sarkar ${ }^{50}$ do show two large bands that could be partly related to $\mathrm{H}$-bonding between monomer units besides the larger contribution of $\mathrm{OH}$ groups 


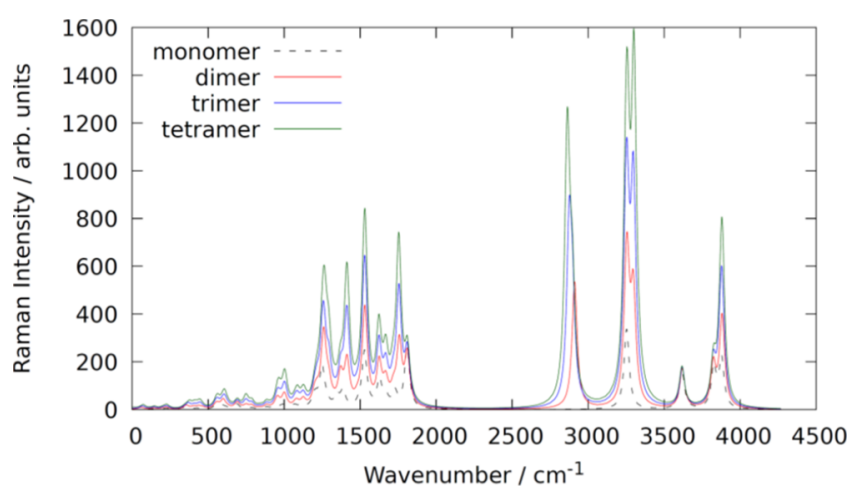

Figure 11. Raman spectra for the most stable dimeric (red), trimeric (blue), and tetrameric (dark green) aggregates. The monomer spectrum (dashed line) is reported as a reference.

typically found in this vibrational range that hampers the Raman analysis of CZA water solution down to $3500 \mathrm{~cm}^{-1}$. In a very recent paper, we showed that, besides the formation of different ionic species, aggregation is also important for the magnetic features of CZA-related carbon nanodots. ${ }^{51}$

3.6. Raman and IR Spectra. To experimentally test the formation of aggregates, we performed SERS measurements of a concentrated water solution of CZA dropped on Ag-coated ITO substrates. After solvent evaporation, we obtained a Raman spectrum in very good agreement with the vibrational features previously reported ${ }^{50}$ and with the calculated modes, with most intense vibrations recorded in the $1200-1800 \mathrm{~cm}^{-1}$ range (Figure 12 and Table $S 1$ in the Supporting Information).

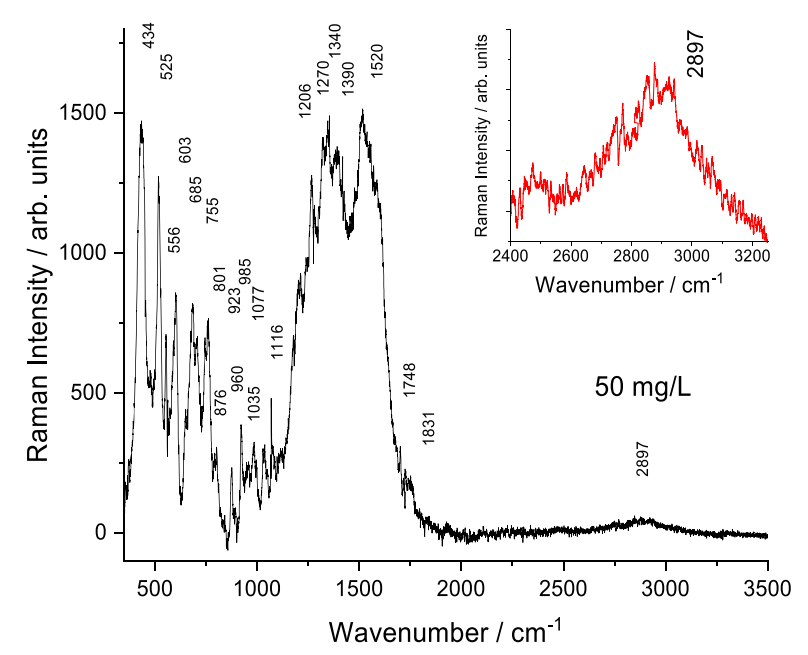

Figure 12. Experimental SERS spectrum of $50 \mathrm{mg} / \mathrm{L}$ CZA water solution. The inset shows a magnification in the $2400-3300 \mathrm{~cm}^{-1}$ range.

In addition to the main modes, the recorded spectrum shows the presence of a vibrational band at $2900 \mathrm{~cm}^{-1}$ that could be ascribed to $\mathrm{H}$-bonding interaction (see the inset of Figure 12), thus fully confirming the calculated spectra and the formation of aggregates.

It is finally important to underline that, besides the observation of the aggregation fingerprints, the formation of the aggregates causes a small intensity increase in the calculated vibrational features as compared to the ones of the monomer structure (from a factor 2 to 3 from dimers to tetramers). Vibrations are responsible, in general, for non- radiative relaxation from the excited state and cause the molecular emission to be independent of excitation. The calculated IR intensities in oligomers could help to explain why, if we assume aggregation as one of the main mechanisms for the widening of the absorption spectrum of CDs down to a visible range, they still have a quite high quantum yield and display excitation-dependent emission properties. ${ }^{52}$

\section{CONCLUSIONS}

The topic of molecular aggregation is dramatically relevant for bottom-up synthesized CDs where molecule-like models are considered as the most reliable model for the observed optical properties. Indeed, spectral tuning can be achieved by engineering the precursors and their concentration. Among the others, CZA occupies a prominent position because citric acid and urea are frequently used to prepare efficient CDs. We demonstrated that CZA can form in-line aggregates whose spectral features can account for the measured shift and widening of the UV $340 \mathrm{~nm}$ absorption band. The aggregation is mediated by $\mathrm{H}$-bonding interaction and to properly deal with $\mathrm{H}$-bonding the application of a proper long-range functional was essential to achieve minimum energy structures. Aggregation was also verified by vibrational spectra, experimental SERS data, and calculated one, being in very good agreement and both showing a fingerprint mode at about 2900 $\mathrm{cm}^{-1}$. NTO analysis assigned an $\mathrm{n}-\pi^{*}$ character and a $\pi-\pi^{*}$ character to the UV and deep UV absorption transitions.

Although these results can be properly referred only to the CZA-related CDs, they can help in the general interpretation of the observed features of bottom-up prepared CDs, promoting the molecular model and the aggregation phenomena as an efficient and persuasive explanation of their optical and vibrational properties and endorsing a closer collaboration between experimentalist and computational theorist researchers in finding the most meaningful correlation between CDs' nanometric structure and chemical-physics properties.

\section{ASSOCIATED CONTENT}

Supporting Information

The Supporting Information is available free of charge at https://pubs.acs.org/doi/10.1021/acs.jpcc.0c10916.

Deconvolution of absorption bands, experimental and calculated vibrations, NTOs of trimers and tetramers, and calculated IR spectra (PDF)

\section{AUTHOR INFORMATION}

\section{Corresponding Author}

Carlo M. Carbonaro - Department of Physics, Univ. of Cagliari, Cittadella Universitaria, Monserrato 09042, Italy; () orcid.org/0000-0001-6353-6409;

Email: cm.carbonaro@dsf.unica.it

\section{Authors}

Antonio Cappai - Department of Physics, Univ. of Cagliari, Cittadella Universitaria, Monserrato 09042, Italy; (1) orcid.org/0000-0002-8415-778X

Claudio Melis - Department of Physics, Univ. of Cagliari, Cittadella Universitaria, Monserrato 09042, Italy; (1) orcid.org/0000-0002-5768-8403

Luigi Stagi - Department of Chemistry and Pharmacy, Laboratory of Materials Science and Nanotechonology, CR- 
INSTM, Sassari 07100, Italy; @ orcid.org/0000-00027238-8425

Pier C. Ricci - Department of Physics, Univ. of Cagliari, Cittadella Universitaria, Monserrato 09042, Italy; (1) orcid.org/0000-0001-6191-4613

Francesca Mocci - Department of Chemical and Geological Sciences, University of Cagliari, Monserrato I-09042, Italy; (1) orcid.org/0000-0003-1394-9146

Complete contact information is available at: https://pubs.acs.org/10.1021/acs.jpcc.0c10916

\section{Author Contributions}

The manuscript was written through contributions of all authors.

\section{Funding}

Italian Ministry of University and Research (MIUR) within the project PRIN2017 "CANDL2" (Grant 2017W75RAE) and "Fondazione di Sardegna" within the project L.R 7. CUP F74I19000930007 "NG-Light: a new generation of phosphors" are gratefully acknowledged.

\section{Notes}

The authors declare no competing financial interest.

\section{ACKNOWLEDGMENTS}

MUR and Fondazione di Sardegna are gratefully acknowledged.

\section{REFERENCES}

(1) Xu, X.; Ray, R.; Gu, Y.; Ploehn, H. J.; Gearheart, L.; Raker, K.; Scrivens, W. A. Electrophoretic Analysis and Purification of Fluorescent Single-Walled Carbon Nanotube Fragments. J. Am. Chem. Soc. 2004, 126, 12736-12737.

(2) Joseph, J.; Anappara, A. A. White light emission of carbon dots by creating different emissive traps. J. Lumin. 2016, 178, 128-133.

(3) Kang, Y.-F.; Li, Y.-H.; Fang, Y.-W.; Xu, Y.; Wei, X.-M.; Yin, X.-B. Carbon Quantum Dots for Zebrafish Fluorescence Imaging. Sci. Rep. 2015, 5, 11835.

(4) Chen, H.; Xie, Y.; Kirillov, A. M.; Liu, L.; Yu, M.; Liu, W.; Tang, Y. A ratiometric fluorescent nanoprobe based on terbium functionalized carbon dots for highly sensitive detection of an anthrax biomarker. Chem. Commun. 2015, 51, 5036-5039.

(5) Bhunia, S. K.; Maity, A. R.; Nandi, S.; Stepensky, D.; Jelinek, R. Imaging Cancer Cells Expressing the Folate Receptor with Carbon Dots Produced from Folic Acid. ChemBioChem 2016, 17, 614-619.

(6) Zhu, S.; Meng, Q.; Wang, L.; Zhang, J.; Song, Y.; Jin, H.; Zhang, K.; Sun, H.; Wang, H.; Yang, B. Highly Photoluminescent Carbon Dots for Multicolor Patterning, Sensors, and Bioimaging. Angew. Chem., Int. Ed. 2013, 52, 3953-3957.

(7) Shen, L.; Chen, M.; Hu, L.; Chen, X.; Wang, J. Growth and Stabilization of Silver Nanoparticles on Carbon Dots and Sensing Application. Langmuir 2013, 29, 16135-16140.

(8) Yu, H.; Shi, R.; Zhao, Y.; Waterhouse, G. I. N.; Wu, L.-Z.; Tung, C.-H.; Zhang, T. Smart utilization of carbon dots in semiconductor photocatalysis. Adv. Mater. 2016, 28, 9454-9477.

(9) Han, M.; Zhu, S.; Lu, S.; Song, Y.; Feng, T.; Tao, S.; Liu, J.; Yang, B. Recent progress on the photocatalysis of carbon dots: Classification, mechanism and applications. Nano Today 2018, 19, 201-218.

(10) Schneider, J.; Reckmeier, C. J.; Xiong, Y.; von Seckendor, M.; Susha, A. S.; Kasak, P.; Rogach, A. L. Molecular Fluorescence in Citric Acid-Based Carbon Dots. J. Phys. Chem. C 2017, 121, 2014-2022.

(11) Kasprzyk, W.; Swiergosz, T.; Bednarz, S.; Walas, K.; Bashmakova, N. V.; Bogdal, D. Luminescence phenomena of carbon dots derived from citric acid and urea - a molecular insight. Nanoscale 2018, 10, 13889-13894.
(12) Song, Y.; Zhu, S.; Zhang, S.; Fu, Y.; Wang, L.; Zhao, X.; Yang, $B$. Investigation from chemical structure to photoluminescent mechanism: a type of carbon dots from the pyrolysis of citric acid and an amine. J. Mater. Chem. C 2015, 3, 5976-5984.

(13) Carbonaro, C. M.; Corpino, R.; Salis, M.; Mocci, F.; Thakkar, S. V.; Olla, C.; Ricci, P. C. On the Emission Properties of Carbon Dots: Reviewing Data and Discussing Models. J. Carbon Res. 2019, 5, 60.

(14) Margraf, J. T.; Strauss, V.; Guldi, D. M.; Clark, T. The Electronic Structure of Amorphous Carbon Nanodots. J. Phys. Chem. B 2015, 119, 7258-7265.

(15) Hola, K.; Sudolska, M.; Kalytchuk, S.; Nachtigallova, D.; Rogach, A. L.; Otyepka, M.; Zboril, R. Graphitic Nitrogen Triggers Red Fluorescence in Carbon Dots. ACS Nano 2017, 11, 1240212410.

(16) Shamsipur, M.; Barati, A.; Taherpour, A. A.; Jamshidi, M. Resolving the Multiple Emission Centers in Carbon Dots: From Fluorophore Molecular States to Aromatic Domain States and Carbon-Core States. J. Phys. Chem. Lett. 2018, 9, 4189-4198.

(17) Sudolska, M.; Otyepka, M. Exact roles of individual chemical forms of nitrogen in the photoluminescent properties of nitrogendoped carbon dots. Appl. Mater. Today 2017, 7, 190.

(18) Strauss, V.; Margraf, J. T.; Dolle, C.; Butz, B.; Nacken, T. J.; Walter, J.; Bauer, W.; Peukert, W.; Spiecker, E.; Clark, T.; Guldi, D. M. Carbon Nanodots: Toward a Comprehensive Understanding of Their Photoluminescence. J. Am. Chem. Soc. 2014, 136, 1730817316.

(19) Tepliakov, N. V.; Kundelev, E. V.; Khavlyuk, P. D.; Xiong, Y.; Leonov, M. Y.; Zhu, W.; Baranov, A. V.; Fedorov, A. V.; Rogach, A. L.; Rukhlenko, I. D. sp2-sp3-Hybridized Atomic Domains Determine Optical Features of Carbon Dots. ACS Nano 2019, 13, 10737-10744.

(20) Nandy, A.; Kumar, A.; Dwivedi, S.; Pal, S. K.; Panda, D. Connecting the Dots of Carbon Nanodots: Excitation (In)dependency and White-Light Emission in One-Step. J. Phys. Chem. C 2019, 123, 20502-20511.

(21) Palonćyová, M.; Langer, M.; Otyepka, M. Structural Dynamics of Carbon Dots in Water and N,N-Dimethylformamide Probed by All-Atom Molecular Dynamics Simulations. J. Chem. Theory Comput. 2018, 14, 2076-2083.

(22) Siddique, F.; Langer, M.; Paloncyova, M.; Medved, M.; Otyepka, M.; Nachtigallova, D.; Lischka, H.; Aquino, A. J. Molecular Fluorophores Self-Organize into C-Dot Seeds and Incorporate into CDot Structures. J. Phys. Chem. C 2020, 11, 8252-8258.

(23) Kundelev, E. V.; Tepliakov, N. V.; Leonov, M. Y.; Maslov, V. G.; Baranov, A. V.; Fedorov, A. V.; Rukhlenko, I. D.; Rogach, A. L. Toward Bright Red-Emissive Carbon Dots through Controlling Interaction among Surface Emission Centers. J. Phys. Chem. Lett. 2020, 11, 8121--8127.

(24) Sharma, A.; Gadly, T.; Gupta, A.; Ballal, A.; Ghosh, S. K.; Kumbhakar, M. Origin of Excitation Dependent Fluorescence in Carbon Nanodots. J. Phys. Chem. Lett. 2016, 7, 3695-3702.

(25) Sharma, A.; Gadly, T.; Neogy, S.; Ghosh, S. K.; Kumbhakar, M. Molecular Origin and Self-Assembly of Fluorescent Carbon Nanodots in Polar Solvents. J. Phys. Chem. Lett. 2017, 8, 1044-1052.

(26) Reckmeier, C. J.; Schneider, J.; Xiong, Y.; Hausler, J.; Kasak, P.; Schnick, W.; Rogach, A. L. Aggregated molecular fluorophores in the ammonothermal synthesis of carbon dots. Chem. Mater. 2017, 29, 10352-10361.

(27) Ludmerczki, R.; Mura, S.; Carbonaro, C. M.; Mandity, I. M.; Carraro, M.; Senes, N.; Garroni, S.; Granozzi, G.; Calvillo, L.; Marras, S.; Malfatti, L.; Innocenzi, P. Carbon Dots from Citric Acid and its Intermediates Formed by Thermal Decomposition. Chem. - Eur. J. 2019, 25, 11963-11974.

(28) Mintz, K. J.; Zhou, Y.; Leblanc, R. M. Recent development of carbon quantum dots regarding their optical properties, photoluminescence mechanism, and core structure. Nanoscale 2019, 11, 4634-4652.

(29) Carbonaro, C. M.; Chiriu, D.; Stagi, L.; Casula, M. F.; Thakkar, S. V.; Malfatti, L.; Suzuki, K.; Ricci, P. C.; Corpino, R. Carbon Dots in 
Water and Mesoporous Matrix: Chasing the Origin of their Photoluminescence. J. Phys. Chem. C 2018, 122, 25638-25650.

(30) Mura, S.; Stagi, L.; Malfatti, L.; Carbonaro, C. M.; Ludmerczki, R.; Innocenzi, P. Modulating the Optical Properties of Citrazinic Acid through the Monomer-to-Dimer Transformation. J. Phys. Chem. A 2019, 124, 197-203.

(31) Mura, S.; Ludmerczki, R.; Stagi, L.; Garroni, S.; Carbonaro, C. M.; Ricci, P. C.; Casula, M. F.; Malfatti, L.; Innocenzi, P. Integrating sol-gel and carbon dots chemistry for the fabrication of fluorescent hybrid organic-inorganic films. Sci. Rep. 2020, 10, 4770.

(32) Szyc, L.; Yang, M.; Nibbering, E.; Elsaesser, T. Ultrafast vibrational dynamics and local interactions of hydrated DNA. Angew. Chem., Int. Ed. 2010, 49, 3598-3610.

(33) Boese, A. D. Density functional theory and hydrogen bonds: are we there yet? ChemPhysChem 2015, 16, 978-985.

(34) Chai, J.-D.; Head-Gordon, M. Long-range corrected hybrid density functionals with damped atom-atom dispersion corrections. Phys. Chem. Chem. Phys. 2008, 10, 6615-6620.

(35) Chai, J.-D.; Head-Gordon, M. Systematic optimization of longrange corrected hybrid density functionals. J. Chem. Phys. 2008, 128, 084106.

(36) Jacquemin, D.; Perpete, E. A.; Ciofini, I.; Adamo, C. Assessment of the $\omega \mathrm{B} 97$ family for excited-state calculations. Theor. Chem. Acc. 2011, 128, 127-136.

(37) Burns, L. A.; Mayagoitia, A. V.; Sumpter, B. G.; Sherrill, C. D. Density-functional approaches to noncovalent interactions: A comparison of dispersion corrections (DFT-D), exchange-hole dipole moment (XDM) theory, and specialized functionals. J. Chem. Phys. 2011, 134, 084107.

(38) Kasha, M.; Rawls, H. R.; El-Bayoumi, M. A. The exciton model in molecular spectroscopy. Pure Appl. Chem. 1965, 11, 371-392.

(39) Anedda, A.; Carbonaro, C. M.; Corpino, R.; Ricci, P. C.; Grandi, S.; Mustarelli, P. Formation of fluorescent aggregates in Rhodamine 6G doped silica glasses. J. Non-Cryst. Solids 2007, 353, 481.

(40) Frisch, M. J.; Trucks, G. W.; Schlegel, H. B.; Scuseria, G. E.; Robb, M. A.; Cheeseman, J. R.; Scalmani, G.; Barone, V.; Petersson, G. A.; Nakatsuji, H.; Li, X.; Caricato, M.; Marenich, A. V.; Bloino, J.; Janesko, B. G.; Gomperts, R.; Mennucci, B.; Hratchian, H. P.; Ortiz, J. V.; Izmaylov, A. F.; Sonnenberg, J. L.; Williams-Young, D.; Ding, F.; Lipparini, F.; Egidi, F.; Goings, J.; Peng, B.; Petrone, A.; Henderson, T.; Ranasinghe, D.; Zakrzewski, V. G.; Gao, J.; Rega, N.; Zheng, G.; Liang, W.; Hada, M.; Ehara, M.; Toyota, K.; Fukuda, R.; Hasegawa, J.; Ishida, M.; Nakajima, T.; Honda, Y.; Kitao, O.; Nakai, H.; Vreven, T.; Throssell, K.; Montgomery, J. A.; Peralta, J. E.; Ogliaro, F.; Bearpark, M. J.; Heyd, J. J.; Brothers, E. N.; Kudin, K. N.; Staroverov, V. N.; Keith, T. A.; Kobayashi, R.; Normand, J.; Raghavachari; Rendell, A. P.; Burant, J. C.; Iyengar, S. S.; Tomasi, J.; Cossi, M.; Millam, J. M.; Klene, M.; Adamo, C.; Cammi, R.; Ochterski, J. W.; Martin, R. L.; Morokuma, K.; Farkas, O.; Foresman, J. B.; Fox, D. J. Gaussian 16 revision c.01 2016, Gaussian Inc. Wallingford CT.

(41) Becke, A. D. Density-functional thermochemistry. III. The role of exact exchange. J. Chem. Phys. 1993, 98, 5648-5652.

(42) Tirado-Rives, J.; Jorgensen, W. L. Performance of B3LYP Density Functional Methods for a Large Set of Organic Molecules. J. Chem. Theory Comput. 2008, 4, 297-306.

(43) Cancès, E.; Mennucci, B.; Tomasi, J. A new integral equation formalism for the polarizable continuum model: Theoretical background and applications to isotropic and anisotropic dielectrics. J. Chem. Phys. 1997, 107, 3032-3041.

(44) Carbonaro, C. M. Tuning the formation of aggregates in silicaRhodamine 6G hybrids by thermal treatment. J. Photochem. Photobiol., A 2011, 222, 56-63.

(45) Carbonaro, C. M.; Orrù, F.; Ricci, P. C.; Ardu, A.; Corpino, R.; Chiriu, D.; Angius, F.; Mura, A.; Cannas, C. High efficient fluorescent stable colloidal sealed dye-doped mesostructured silica nanoparticles. Microporous Mesoporous Mater. 2016, 225, 432-439.

(46) Stagi, L.; Mura, S.; Malfatti, L.; Carbonaro, C. M.; Ricci, P. C.; Porcu, S.; Secci, F.; Innocenzi, P. Anomalous Optical Properties of
Citrazinic Acid under Extreme pH Conditions. ACS Omega 2020, 5, 10958-10964.

(47) Martin, R. L. Natural transition orbitals. J. Chem. Phys. 2003, 118, 4775.

(48) Kasprzyk, W.; Bednarz, S.; Zmudzki, P.; Galica, M.; Bogdal, D. Novel efficient fluorophores synthesized from citric acid. RSC $A d v$. 2015, 5, 34795-34799.

(49) Sudolska, M.; Dubecky, M.; Sarkar, S.; Reckmeier, C. J.; Zboril, R.; Rogach, A. L.; Otyepka, M. Nature of Absorption Bands in Oxygen-Functionalized Graphitic Carbon Dots. J. Phys. Chem. C 2015, 119, 13369-13373.

(50) Sarkar, S.; Sudolska, M.; Dubecky, M.; Reckmeier, C. J.; Rogach, A. L.; Zboril, R.; Otyepka, M. Graphitic Nitrogen Doping in Carbon Dots Causes Red-Shifted Absorption. J. Phys. Chem. C 2016, 120, 1303-1308.

(51) Mocci, F.; Olla, C.; Cappai, A.; Corpino, R.; Ricci, P. C.; Chiriu, D.; Salis, M.; Carbonaro, C. M. Formation of Citrazinic Acid Ions and Their Contribution to Optical and Magnetic Features of Carbon Nanodots: A Combined Experimental and Computational Approach. Materials 2021, 14, 770.

(52) Zhang, J.; Yang, L.; Yuan, Y.; Jiang, J.; Yu, S.-H. One-Pot GramScale Synthesis of Nitrogen and Sulfur Embedded Organic Dots with Distinctive Fluorescence Behaviors in Free and Aggregated States. Chem. Mater. 2016, 28, 4367-4374. 